\title{
A Well-Balanced Unified Gas-Kinetic Scheme for Multiscale Flow Transport Under Gravitational Field
}

\author{
Tianbai Xiao ${ }^{\mathrm{a}}$, Qingdong Cai ${ }^{\mathrm{a}}$, Kun Xu $\mathrm{u}^{\mathrm{b}, *}$ \\ ${ }^{a}$ Department of Mechanics and Engineering Science, College of Engineering, Peking University, Beijing \\ 100871, China \\ ${ }^{b}$ Department of Mathematics, Department of Mechanical and Aerospace Engineering, Hong Kong \\ University of Science and Technology, Clear Water Bay, Kowloon, Hong Kong
}

\begin{abstract}
The gas dynamics under gravitational field is usually associated with multiple scale nature due to large density variation and a wide variation of local Knudsen number. It is challenging to construct a reliable numerical algorithm to accurately capture the nonequilibrium physical effect in different regimes. In this paper, a well-balanced unified gaskinetic scheme (UGKS) for all flow regimes under gravitational field will be developed, which can be used for the study of non-equilibrium gravitational gas system. The well-balanced scheme here is defined as a method to evolve an isolated gravitational system under any initial condition to a hydrostatic equilibrium state and to keep such a solution. To preserve such a property is important for a numerical scheme, which can be used for the study of slowly evolving gravitational system, such as the formation of star and galaxy. Based on the Boltzmann model with external forcing term, the UGKS uses an analytic time-dependent (or scale-dependent) solution in the construction of the discretized fluid dynamic equations in the cell size and time step scales, i.e., the so-called direct modeling method. As a result, with the variation of the ratio between the numerical time step and local particle collision time, the UGKS is able to recover flow physics in different regimes and provides a continuous spectrum of gas dynamics. For the first time, the flow physics of a gravitational system in the transition regime can be studied using the UGKS, and the non-equilibrium phenomena in such a gravitational system can be clearly identified. Many numerical examples will be used to validate the scheme. New physical observation, such as the correlation between the gravitational field and the heat flux in the transition regime, will be presented. The current method provides an indispensable tool for the study of non-equilibrium gravitational system.
\end{abstract}

Keywords: gravitational field, well-balanced property, unified gas-kinetic scheme, multi-scale flow, non-equilibrium phenomena

\footnotetext{
*Corresponding author

Email addresses: xiaotianbai@pku.edu.cn (Tianbai Xiao), caiqd@pku.edu.cn (Qingdong Cai), makxu@ust.hk (Kun Xu)
}

Preprint submitted to Elsevier

December 13, 2016 


\section{Introduction}

The universe is an evolving gravitational system. The gas dynamics due to the gravitational force plays a critical role in the star and galaxy formation, as well as atmospheric convection on the planets. For an evolving gravitational system, there is almost no any validated governing equation to describe the non-equilibrium dynamics uniformly across different regimes. The well-established statistical mechanics is mostly for the equilibrium solution. For an isolated gravitational system, under the conservation of mass, momentum, and energy, the system will eventually get to the hydrostatic equilibrium state from an arbitrary initial condition. Such a steady-state solution will be maintained due to exact balance between the gravitational source term and the inhomogeneous flux function. For the study of a gravitational system, many numerical schemes have been developed. To capture such an equilibrium solution for an isolated gravitational system is a minimal requirement for a scheme which can be used in the astrophysical applications. The lack of well-balanced property may lead to spurious solution, or even present wrong physical solution for a long time evolving gravitational system. The purpose of this paper is to develop such a scheme which not only has the well-balanced property, but also be able to capture the non-equilibrium phenomena, which have not been studied theoretically or numerically for a gravitational system before.

For the equilibrium flow, such as for the gravitational Euler system, many efforts have been devoted to the construction of well-balanced schemes. Leveque and Bale [1] developed a quasi-steady wave-propagation algorithm, which is able to capture perturbed quasi-steady solutions. Botta et al. [2] used local, time dependent hydrostatic reconstructions to achieve the hydrostatic balance. Xing and Shu [3] proposed a high-order WENO scheme to resolve small perturbations on the hydrostatic balance state on coarse meshes. The basic idea of these methods is to extend classical algorithm for the compressible Euler equations to the gravitational system with proper modifications.

Different gas dynamic equations can be constructed under different modeling scales. In the kinetic scale, such as particle mean free path and traveling time between particle collisions, the Boltzmann equation is well established. In the hydrodynamic scale, even though the scale for its modeling is not clearly identified, the Euler and Navier-Stokes equations are routinely used. Due to the clear scale separation, both kinetic and hydrodynamic equations can be applied in their respective scales. However, the real gas dynamics may not have such a scale separation. With the scale variation between the kinetic and hydrodynamic ones, the gas dynamic equation should have a smooth transition from the Boltzmann to the Navier-Stokes equations. This multiple scale nature is especially important for a gravitational system, where due to the gravitational effect the density in the system can be varied largely, and so is the particle mean free path. With a fixed modeling scale, such as the mesh size in a numerical scheme, the cell's Knudsen number $\mathrm{Kn}_{\mathrm{c}}$ can be changed significantly. For a single gravitational system, the flow dynamics may vary from the kinetic Boltzmann modeling in the upper atmospheric layer to the hydrodynamic one in the inner high density region, with a continuous variation of flow physics. Therefore, a gravitational system has an intrinsic multiple scale nature. The corresponding numerical algorithm to simulate such a 
system is preferable to have a property of providing a continuous spectrum of flow dynamics from rarefied to continuum one.

In recent years, the unified gas-kinetic scheme has been developed for the simulation of multiple scale flow problems $[4,5,6,7]$. This algorithm is based on the direct physical modeling on the mesh size scale, such as constructing the corresponding governing equations in such a scale. The scheme is able to capture physical solution in all flow regimes. The coupled treatment of particle transport and collision in the evaluation of a time-dependent interface flux function is the key for its cross-scale modeling and ensures a multiple scale nature of the algorithm. The mechanism of flow evolution in different regimes is determined by the ratio between the time step and the local particle collision time. In this paper, the further development of the scheme for a gravitational system is proposed.

In order to develop a well-balanced gas-kinetic scheme, the most important ingredient is to take the external force effect into the flux transport across a cell interface. Attempts have been made in the construction of the schemes for the shallow water equations [8] and gas dynamic equations $[9,10,11]$. In this paper, the similar methodology is used in the unified gas-kinetic scheme. The scheme can be used to study the multiple scale non-equilibrium flow phenomena under gravitational field, which has never been fully explored before.

This paper is organized as following. Section 2 is about the kinetic theory under gravitational field. Section 3 presents the construction of the unified gas-kinetic scheme for a gravitational system. Section 4 includes numerical examples to demonstrate the performance of the scheme. The last section is the conclusion.

\section{Gas kinetic modeling}

The gas kinetic theory describes the evolution of particle distribution function $f\left(x_{i}, t, u_{i}, \xi\right)$ in space and time $\left(x_{i}, t\right)$. Here $u_{i}=(u, v, w)$ is particle velocity and $\xi$ is the internal variable for the rotation and vibration. The evolution equation for $f$ in the kinetic scale with the separate modeling of particle transport and collision is the so-called Boltzmann equation,

$$
f_{t}+u_{i} f_{x_{i}}+\phi_{i} f_{u_{i}}=Q(f, f),
$$

where $\phi_{i}$ is the external forcing term and $Q(f, f)$ is the collision term.

Many kinetic models, such as BGK [12], ES-BGK [13], Shakhov [14], and even the full Boltzmann equation [15], can be used in the construction of the unified gas-kinetic scheme. Here we use the model equation to explain the principle for the algorithm development. The generalized one-dimensional BGK-type model with the inclusion of external force $\phi_{x}$ can be written as

$$
f_{t}+u f_{x}+\phi_{x} f_{u}=\frac{f^{+}-f}{\tau}
$$

where $f^{+}$is the equilibrium state, and $\tau$ is the collision time. For the BGK equation, $f^{+}$is exactly the Maxwellian distribution

$$
f^{+}=g_{0}=\rho\left(\frac{\lambda}{\pi}\right)^{\frac{K+1}{2}} e^{-\lambda\left[(u-U)^{2}+\xi^{2}\right]},
$$


where $\lambda=\rho /(2 p)$ and $K$ is the dimension of $\xi$. For the Shakhov model equation, $f^{+}$takes the form,

$$
f^{+}=g_{0}\left[1+(1-\operatorname{Pr}) c q\left(\frac{c^{2}}{R T}-5\right) /(5 p R T)\right]
$$

where $c=u-U$ is the peculiar velocity, $q$ is heat flux, and $\operatorname{Pr}$ is Prandtl number. The collision term satisfies the compatibility condition

$$
\int\left(f^{+}-f\right) \psi d \Xi=0
$$

where $\psi=\left(1, u, \frac{1}{2}\left(u^{2}+\xi^{2}\right)\right)^{T}$ is a vector of moments for collision invariants, and $d \Xi=d u d \xi$. The macroscopic conservative flow variables are the moments of the particle distribution function via

$$
\mathbf{W}=\left(\begin{array}{c}
\rho \\
\rho U \\
\rho E
\end{array}\right)=\int f \psi d \Xi .
$$

With a local constant collision time $\tau$, the integral solution of Eq.(1) can be constructed by the method of characteristics,

$$
\begin{aligned}
f(x, t, u, \xi)= & \frac{1}{\tau} \int_{t^{n}}^{t} f^{+}\left(x^{\prime}, t^{\prime}, u^{\prime}, \xi\right) e^{-\left(t-t^{\prime}\right) / \tau} d t^{\prime} \\
& +e^{-\left(t-t^{n}\right) / \tau} f_{0}^{n}\left(x^{n}, t^{n}, u^{n}, \xi\right),
\end{aligned}
$$

where $x^{\prime}=x-u^{\prime}\left(t-t^{\prime}\right)-\frac{1}{2} \phi_{x}\left(t-t^{\prime}\right)^{2}$ and $u^{\prime}=u-\phi_{x}\left(t-t^{\prime}\right)$ are the trajectories in physical and phase space, and $f_{0}^{n}$ is the gas distribution function at the beginning of $n$-th time step. The above integral solution plays the most important role for the construction of the well-balanced UGKS.

\section{Numerical algorithm}

\subsection{Construction of interface distribution function}

In the unified scheme, the distribution function $f\left(x_{i+1 / 2}, t, u_{k}, \xi\right)$ at the cell interface $x_{i+1 / 2}$ is constructed from the evolution solution Eq.(2) for the interface flux evaluation. With the notation of $x_{i+1 / 2}=0$ and $t^{n}=0$, the time-dependent interface distribution function becomes

$$
\begin{aligned}
f\left(0, t, u_{k}, \xi\right)= & \frac{1}{\tau} \int_{0}^{t} f^{+}\left(x^{\prime}, t^{\prime}, u_{k}^{\prime}, \xi\right) e^{-\left(t-t^{\prime}\right) / \tau} d t^{\prime} \\
& +e^{-t / \tau} f_{0}\left(x^{0}, 0, u_{k}^{0}, \xi\right),
\end{aligned}
$$

where $\left(x^{0}, u^{0}\right)$ is the initial location in physical and velocity space for the particle which passes through the cell interface at time $t$. Based on the above integral solution with particle acceleration, the well-balanced algorithm can be constructed similarly as the original UGKS. 
Note that time accumulating effect from the external forcing term on the time evolution of the gas distribution function should be explicitly taken into account.

To the second-order accuracy, the initial gas distribution function $f_{0}$ around the cell interface $x_{i+1 / 2}$ is reconstructed as

$$
f_{0}\left(x, 0, u_{k}, \xi\right)=\left\{\begin{array}{l}
f_{i+1 / 2, k}^{L}+\sigma_{i, k} x, \quad x \leq 0, \\
f_{i+1 / 2, k}^{R}+\sigma_{i+1, k} x, \quad x>0,
\end{array}\right.
$$

where $f_{i+1 / 2, k}^{L}$ and $f_{i+1 / 2, k}^{R}$ are the reconstructed initial distribution functions at the left and right hand sides of the cell interface. In the current scheme, the van Leer limiter is used in the reconstruction. The slope of $f$ at the $i$-th cell and $k$-th particle velocity is

$$
\sigma_{i, j}=\left(\operatorname{sign}\left(s_{1}\right)+\operatorname{sign}\left(s_{2}\right)\right) \frac{\left|s_{1}\right|\left|s_{2}\right|}{\left|s_{1}\right|+\left|s_{2}\right|},
$$

where $s_{1}=\left(f_{i, k}-f_{i-1, k}\right) /\left(x_{i}-x_{i-1}\right), s_{2}=\left(f_{i+1, k}-f_{i, k}\right) /\left(x_{i+1}-x_{i}\right)$. The equilibrium distribution function around a cell interface is approximated locally through a Taylor expansion in space and time as

$$
g=g_{0}\left[1+(1-H[x]) a^{L} x+H[x] a^{R} x+A t\right],
$$

where $g_{0}$ is the Maxwellian distribution at $(x=0, t=0)$, and $H[x]$ is the Heaviside step function. Here $a^{L}, a^{R}$, and $A$, are from the Taylor expansion of a Maxwellian,

$$
\begin{aligned}
& a^{L, R}=a_{1}^{L, R}+a_{2}^{L, R} u+a_{3}^{L, R} \frac{1}{2}\left(u^{2}+\xi^{2}\right)=a_{\alpha}^{L, R} \psi_{\alpha}, \\
& A=A_{1}+A_{2} u+A_{3} \frac{1}{2}\left(u^{2}+\xi^{2}\right)=A_{\alpha} \psi_{\alpha} .
\end{aligned}
$$

Based on the compatibility condition at the cell interface, the equilibrium and the corresponding macroscopic conservative variables $\mathbf{W}_{0}$ can be determined from

$$
\left.\int\left(f^{+}-f\right)\right|_{x=0, t=0} \psi d \Xi=0
$$

where $d \Xi=d u d \xi$, which results

$$
\int g_{0} \psi_{\alpha} d \Xi=\mathbf{W}_{0}=\sum_{u_{k}>0} f_{i+1 / 2, k}^{L} \psi d \Xi+\sum_{u_{k}<0} f_{i+1 / 2, k}^{R} \psi d \Xi .
$$

After the determination of the equilibrium state at the cell interface, its spatial slopes $a^{L}, a^{R}$ can be obtained from the slopes of conservative variables on both sides of a cell interface

$$
\left(\frac{\partial \mathbf{W}}{\partial x}\right)^{L}=\int a^{L} g_{0} \psi d \Xi, \quad\left(\frac{\partial \mathbf{W}}{\partial x}\right)^{R}=\int a^{R} g_{0} \psi d \Xi .
$$


The time derivative $A$ of $g_{0}$ is related to the temporal variation of conservative flow variables

$$
\frac{\partial \mathbf{W}}{\partial t}=\int A g_{0} \psi d \Xi
$$

and it can be calculated via time derivative of the compatibility condition

$$
\left.\frac{d}{d t} \int\left(f^{+}-f\right) \psi d \Xi\right|_{x=0, t=0}=0
$$

With the help of the Euler equation with external forcing term, it gives

$$
-\int u \frac{\partial g}{\partial x} \psi d \Xi-\int \phi_{x} \frac{\partial g}{\partial u} \psi d \Xi=\frac{\partial \mathbf{W}}{\partial t}=\int A g_{0} \psi d \Xi
$$

where the spatial derivative can be constructed from the Taylor expansion of equilibrium distribution Eq.(5), and the velocity derivative is obtained from the exact Maxwellian distribution. The result is

$$
-\int a^{L, R} u g_{0} \psi d \Xi+2 \int \phi_{x} \lambda_{0} g_{0}\left(u-U_{0}\right) \psi d \Xi=\frac{\partial \mathbf{W}}{\partial t}=\int A g_{0} \psi d \Xi
$$

where $U_{0}$ and $\lambda_{0}$ are the corresponding macroscopic variables in the equilibrium state $g_{0}$. Using the equation above, we can obtain coefficients $A=\left(A_{1}, A_{2}, A_{3}\right)^{T}$.

After the determination of all coefficients, the time dependent interface distribution function becomes

$$
\begin{aligned}
f\left(0, t, u_{k}, \xi\right)= & \left(1-e^{-t / \tau}\right)\left(g_{0}+g^{+}\right) \\
& +\left(\tau\left(-1+e^{-t / \tau}\right)+t e^{-t / \tau}\right) a^{L, R} u_{k} g_{0} \\
& -\left[\tau\left(\tau\left(-1+e^{-t / \tau}\right)+t e^{-t / \tau}\right)+\frac{1}{2} t^{2} e^{-t / \tau}\right] a^{L, R} \phi_{x} g_{0} \\
& +\tau\left(t / \tau-1+e^{-t / \tau}\right) A g_{0} \\
& +e^{-t / \tau}\left[\left(f_{i+1 / 2, k^{0}}^{L}+\left(-\left(u_{k}-\phi_{x} t\right) t-\frac{1}{2} \phi_{x} t^{2}\right) \sigma_{i, k^{0}}\right) H\left[u_{k}-\frac{1}{2} \phi_{x} t\right]\right. \\
& \left.+\left(f_{i+1 / 2, k^{0}}^{R}+\left(-\left(u_{k}-\phi_{x} t\right) t-\frac{1}{2} \phi_{x} t^{2}\right) \sigma_{i+1, k^{0}}\right)\left(1-H\left[u_{k}-\frac{1}{2} \phi_{x} t\right]\right)\right] \\
= & \widetilde{g}_{i+1 / 2, k}+\widetilde{f}_{i+1 / 2, k},
\end{aligned}
$$

where $\widetilde{g}_{i+1 / 2, k}$ is the first four terms related to equilibrium state integration and $\widetilde{f}_{i+1 / 2, k}$ is the last term from the initial non-equilibrium distribution.

\subsection{Two dimensional case}

The unified gas-kinetic scheme is a multidimensional method, where both derivatives of flow variables in the normal and tangential directions of a cell interface are taken into 
account. With the external force $\vec{\phi}=\phi_{x} \vec{i}+\phi_{y} \vec{j}$, the BGK-type model in the two-dimensional Cartesian coordinate system is

$$
f_{t}+u f_{x}+v f_{y}+\phi_{x} f_{u}+\phi_{y} f_{v}=\frac{f^{+}-f}{\tau}
$$

where $\tau=\mu / p$ is the particle collision time and $f^{+}$is the equilibrium distribution.

The integral solution can be written as

$$
\begin{aligned}
f(x, y, t, u, v, \xi)= & \frac{1}{\tau} \int_{t^{n}}^{t} f^{+}\left(x^{\prime}, y^{\prime}, t^{\prime}, u^{\prime}, v^{\prime}, \xi\right) e^{-\left(t-t^{\prime}\right) / \tau} d t^{\prime} \\
& +e^{-\left(t-t^{n}\right) / \tau} f_{0}^{n}\left(x^{n}, y^{n}, t^{n}, u^{n}, v^{n}, \xi\right)
\end{aligned}
$$

where $x^{\prime}=x-u^{\prime}\left(t-t^{\prime}\right)-\frac{1}{2} \phi_{x}\left(t-t^{\prime}\right)^{2}, y^{\prime}=y-v^{\prime}\left(t-t^{\prime}\right)-\frac{1}{2} \phi_{y}\left(t-t^{\prime}\right)^{2}, u^{\prime}=u-\phi_{x}\left(t-t^{\prime}\right)$, and $v^{\prime}=v-\phi_{y}\left(t-t^{\prime}\right)$.

In the unified scheme, at the center of a cell interface $\left(x_{i+1 / 2}, y_{j}\right)$ the solution $f_{i+1 / 2, j, k, l}$ is constructed from the integral solution Eq.(7). With the notations $x_{i+1 / 2}=0, y_{j}=0$ at $t^{n}=0$, the time-dependent interface distribution function goes to

$$
\begin{aligned}
f\left(0,0, t, u_{k}, v_{l}, \xi\right)= & \frac{1}{\tau} \int_{0}^{t} f^{+}\left(x^{\prime}, y^{\prime}, t^{\prime}, u_{k}^{\prime}, v_{l}^{\prime}, \xi\right) e^{-\left(t-t^{\prime}\right) / \tau} d t^{\prime} \\
& +e^{-t / \tau} f_{0}\left(-\left(u_{k}-\phi_{x} t\right) t-\frac{1}{2} \phi_{x} t^{2},-\left(v_{l}-\phi_{y} t\right) t-\frac{1}{2} \phi_{y} t^{2}, 0, u_{k}-\phi_{x} t, v_{l}-\phi_{y} t, \xi\right),
\end{aligned}
$$

where the trajectories are $x^{\prime}=-u_{k}^{\prime}\left(t-t^{\prime}\right)-\frac{1}{2} \phi_{x}\left(t-t^{\prime}\right)^{2}, y^{\prime}=-v_{l}^{\prime}\left(t-t^{\prime}\right)-\frac{1}{2} \phi_{y}\left(t-t^{\prime}\right)^{2}, u_{k}^{\prime}=$ $u_{k}-\phi_{x}\left(t-t^{\prime}\right)$, and $v_{l}^{\prime}=v_{l}-\phi_{y}\left(t-t^{\prime}\right)$.

As a second order scheme, the initial gas distribution function $f_{0}$ is reconstructed as

$$
f_{0}\left(x, y, 0, u_{k}, v_{l}, \xi\right)=\left\{\begin{array}{l}
f_{i+1 / 2, j, k, l}^{L}+\sigma_{i, j, k, l} x+\theta_{i, j, k, l} y, \quad x \leq 0, \\
f_{i+1 / 2, j, k, l}^{R}+\sigma_{i+1, j, k, l} x+\theta_{i+1, j, k, l} y, \quad x>0,
\end{array}\right.
$$

where $f_{i+1 / 2, j, k, l}^{L}$ and $f_{i+1 / 2, j, k, l}^{R}$ are the reconstructed initial distribution functions at the left and right hand sides of a cell interface. The slope of $f$ at the $(i, j)$ cell and $(k, l)$ discretized velocity point in $x$-direction and $y$-direction is denoted by $\sigma_{i, j, k, l}$ and $\theta_{i, j, k, l}$.

The equilibrium distribution function around a cell interface is constructed as

$$
g=g_{0}\left[1+(1-H[x]) a^{L} x+H[x] a^{R} x+b y+A t\right],
$$

where $g_{0}$ is the Maxwellian distribution at $(x=0, t=0)$. Here $a^{L}, a^{R}$, and $A$ are from the 
Taylor expansion of a Maxwellian

$$
\begin{aligned}
& a^{L, R}=a_{1}^{L, R}+a_{2}^{L, R} u+a_{3}^{L, R} v+a_{4}^{L, R} \frac{1}{2}\left(u^{2}+v^{2}+\xi^{2}\right)=a_{\alpha}^{L, R} \psi_{\alpha}, \\
& b=b_{1}+b_{2} u+b_{3} v+b_{4} \frac{1}{2}\left(u^{2}+v^{2}+\xi^{2}\right)=b_{\alpha} \psi_{\alpha} \\
& A=A_{1}+A_{2} u+A_{3} v+A_{4} \frac{1}{2}\left(u^{2}+v^{2}+\xi^{2}\right)=A_{\alpha} \psi_{\alpha} .
\end{aligned}
$$

The coefficients above can be determined in the same way as one-dimensional case. The time dependent interface distribution function writes

$$
\begin{aligned}
f\left(0,0, t, u_{k}, v_{l}, \xi\right)= & \left(1-e^{-t / \tau}\right)\left(g_{0}+g^{+}\right) \\
& +\left(\tau\left(-1+e^{-t / \tau}\right)+t e^{-t / \tau}\right) a^{L, R} u_{k} g_{0} \\
& -\left[\tau\left(\tau\left(-1+e^{-t / \tau}\right)+t e^{-t / \tau}\right)+\frac{1}{2} t^{2} e^{-t / \tau}\right] a^{L, R} \phi_{x} g_{0} \\
& +\left(\tau\left(-1+e^{-t / \tau}\right)+t e^{-t / \tau}\right) b v_{l} g_{0}-\left[\tau\left(\tau\left(-1+e^{-t / \tau}\right)+t e^{-t / \tau}\right)+\frac{1}{2} t^{2} e^{-t / \tau}\right] b \phi_{y} g_{0} \\
& +\tau\left(t / \tau-1+e^{-t / \tau}\right) A g_{0} \\
& +e^{-t / \tau}\left[\left(f_{i+1 / 2, k^{0}, l^{0}}^{L}+\left(-\left(u_{k}-\phi_{x} t\right) t-\frac{1}{2} \phi_{x} t^{2}\right) \sigma_{i, k^{0}, l^{0}}\right.\right. \\
& \left.+\left(-\left(v_{l}-\phi_{y} t\right) t-\frac{1}{2} \phi_{y} t^{2}\right) \theta_{i, k^{0}, l^{0}}\right) H\left[u_{k}-\frac{1}{2} \phi_{x} t\right] \\
& +\left(f_{i+1 / 2, k^{0}, l^{0}}^{R}+\left(-\left(u_{k}-\phi_{x} t\right) t-\frac{1}{2} \phi_{x} t^{2}\right) \sigma_{i+1, k^{0}, l^{0}}\right. \\
& \left.\left.+\left(-\left(v_{l}-\phi_{y} t\right) t-\frac{1}{2} \phi_{y} t^{2}\right) \theta_{i+1, k^{0}, l^{0}}\right)\left(1-H\left[u_{k}-\frac{1}{2} \phi_{x} t\right]\right)\right] \\
= & \widetilde{g}_{i+1 / 2, j, k, l}+\widetilde{f}_{i+1 / 2, j, k, l},
\end{aligned}
$$

where $\widetilde{g}_{i+1 / 2, j, k, l}$ is related to equilibrium state integration and $\widetilde{f}_{i+1 / 2, j, k, l}$ is the initial nonequilibrium distribution. The extension of the above method to three dimensional case can be done similarly.

\subsection{Update algorithm}

With the cell averaged distribution function

$$
f_{x_{i}, y_{j}, t^{n}, u_{k}, v_{l}}=f_{i, j, k, l}^{n}=\frac{1}{\Omega_{i, j}(\vec{x}) \Omega_{k, l}(\vec{u})} \int_{\Omega_{i, j}} \int_{\Omega_{k, l}} f\left(x, y, t^{n}, u, v\right) d \vec{x} d \vec{u}
$$


the direct modeling for the flow evolution in a discretized space gives

$$
\begin{aligned}
f_{i, j, k, l}^{n+1}= & f_{i, j, k, l}^{n}+\frac{1}{\Omega_{i, j}} \int_{t^{n}}^{t^{n+1}} \sum_{r=1} u_{r} \hat{f}_{r}(t) \Delta L_{r} d t \\
& +\frac{1}{\Omega_{i, j}} \int_{t^{n}}^{t^{n+1}} \int_{\Omega_{i, j}} Q(f) d \vec{x} d t+\frac{1}{\Omega_{i, j}} \int_{t^{n}}^{t^{n+1}} \int_{\Omega_{i, j}} G(f) d \vec{x} d t
\end{aligned}
$$

where $\hat{f}_{r}$ is the time-dependent gas distribution function at cell interface and $\Delta L_{r}$ is the cell interface length. $Q(f)$ and $G(f)$ are the source terms from collision term and gravitational field,

$$
\begin{aligned}
Q(f) & =\frac{f_{i, j, k, l}^{+}-f_{i, j, k, l}^{n+1 / 2}}{\tau}, \\
G(f) & =-\phi_{x} \frac{\partial}{\partial u} f_{i, j, k, l}^{n+1 / 2}-\phi_{y} \frac{\partial}{\partial v} f_{i, j, k, l}^{n+1 / 2} .
\end{aligned}
$$

In the UGKS, we use the semi-implicit method to model the source term of distribution function

$$
\begin{aligned}
f_{i, j, k, l}^{n+1}= & f_{i, j, k, l}^{n}+\frac{1}{\Omega_{i, j}}\left(F_{i-1 / 2, j, k, l}-F_{i+1 / 2, j, k, l}\right)+\frac{1}{\Omega_{i, j}}\left(F_{i, j-1 / 2, k, l}-F_{i, j+1 / 2, k, l}\right) \\
& +\frac{\Delta t}{2}\left(\frac{f_{i, j, k, l}^{+(n+1)}-f_{i, j, k, l}^{n+1}}{\tau^{n+1}}+\frac{f_{i, j, k, l}^{+(n)}-f_{i, j, k, l}^{n}}{\tau^{n}}\right)-\phi_{x} \Delta t \frac{\partial}{\partial u} f_{i, j, k, l}^{n+1}-\phi_{y} \Delta t \frac{\partial}{\partial v} f_{i, j, k, l}^{n+1} .
\end{aligned}
$$

In order to update the gas distribution function, let's take conservative moments on Eq.(10) first. The updates of the conservation flow variables are

$$
\mathbf{W}_{i, j}^{n+1}=\mathbf{W}_{i, j}^{n}+\frac{1}{\Omega_{i, j}} \int_{t^{n}}^{t^{n+1}} \sum_{r=1} \Delta \mathbf{L}_{r} \cdot \mathbf{F}_{r} d t+\frac{1}{\Omega_{i, j}} \int_{t^{n}}^{t^{n+1}} \int_{\Omega_{i, j}} \mathbf{G}_{i, j} d \vec{x} d t
$$

where $\mathbf{F}_{r}$ are the fluxes of conservative flow variables across the cell interface $\Delta \mathbf{L}_{r}$, and $\mathbf{G}_{i, j}$ is the source term from external force,

$$
\mathbf{G}_{i, j}=\int_{\Omega_{k, l}}\left(-\phi_{x} \Delta t \frac{\partial}{\partial u} f_{i, j, k, l}-\phi_{y} \Delta t \frac{\partial}{\partial v} f_{i, j, k, l}\right) \psi d u d v d \xi .
$$

Eq.(12) can be solved first, and its solution can be used for the construction of the equilibrium state in Eq.(11) at $t^{n+1}$. The gravitational source term of distribution function is solved based on the following principle. For the first point at the edge of the particle velocity space in the downwind direction of the external acceleration, the velocity derivatives of the distribution function at this point is assumed to be zero, and thus there is no gravitational source contribution to update the distribution function at this point. Starting from the next point, the previous updated distribution function at a discretized velocity point is used to determine the velocity derivatives at the next velocity point with an upwind finite difference 
approach. Subsequently, the implicit Eq.(11) for $f^{n+1}$ can be solved explicitly.

In the UGKS, the time step is determined by the CFL condition in both physical and phase space

$$
\Delta t=\mathrm{CFL} \min \left\{\frac{\Delta x \Delta y}{u_{\max } \Delta y+v_{\max } \Delta x}, \frac{\Delta u \Delta v}{\left|\phi_{x}\right| \Delta v+\left|\phi_{y}\right| \Delta u}\right\}
$$

where CFL is the CFL number, and $u_{\max }=\max \left(\left|u_{k}\right|\right), v_{\max }=\max \left(\left|v_{l}\right|\right)$ is the largest discretized particle velocity in $x$ and $y$ directions.

\subsection{Analysis on the well-balanced property}

In the continuum regime, the Euler and Navier-Stokes equations are used to describe macroscopic fluid dynamics. Let's consider one dimensional Euler equations, i.e.,

$$
\begin{aligned}
& \rho_{t}+(\rho U)_{x}=0, \\
& (\rho U)_{t}+\left(\rho U^{2}+p\right)_{x}=\rho \phi_{x} \\
& E_{t}+((E+p) U)_{x}=\rho U \phi_{x} .
\end{aligned}
$$

These equations allow an isothermal hydrostatic solution,

$$
\rho=\rho(x), U=0, p_{x}=\rho \phi_{x}
$$

For a constant gravitational acceleration $\phi_{x}$, the corresponding solution is

$$
\rho=\rho_{0} \exp \left(\frac{\phi_{x} x}{R T}\right), u=0, p=p_{0} \exp \left(\frac{\phi_{x} x}{R T}\right)
$$

where $R$ is the gas constant. Since there is no macroscopic velocity or its derivatives, the Navier-Stokes equations give the same solution as that in Eq.(14). For a well-balanced scheme, such a steady-state solution should be maintained due to the exact balance between gravitational source term and inhomogeneous flux function, i.e.,

$$
\frac{1}{\Delta x} \int_{t^{n}}^{t^{n+1}}\left(F_{i-1 / 2}-F_{i+1 / 2}\right) d t+\frac{1}{\Delta x} \int_{t^{n}}^{t^{n+1}} \int_{x_{1-1 / 2}}^{x_{i+1 / 2}} G_{i} d t=0 .
$$

In the well-balanced unified gas-kinetic scheme, the flux is determined by the distribution function at the cell interface in Eq.(6). In the continuum regime where $\Delta t \gg \tau$, the intensive particle collision will converge the distribution function to

$$
f_{i+1 / 2}=g_{0}+g^{+}-(\tau(a u+A)+t A) g_{0}-\tau^{2} a \phi_{x} g_{0}
$$

We consider the hydrostatic balance and omit the equilibrium correction term $g^{+}$and internal variable $\xi$ for brevity. Then, the interface distribution function reduces to

$$
f_{i+1 / 2}=g_{0}-\tau a u g_{0}-\tau^{2} a \phi_{x} g_{0}
$$


The interface flux is calculated via

$$
\mathbf{F}_{i+1 / 2}=\int u f_{i+1 / 2} \psi d u
$$

Since the interface distribution $f_{i+1 / 2}$ is the combination of Maxwellian, its velocity moments $\int u^{\alpha} g d u=\rho\left\langle u^{\alpha}\right\rangle$ can be evaluated analytically. In the static state, the moments of Maxwellian distribution function are

$$
\begin{aligned}
& <u^{0}>=1, \\
& <u^{1}>=0 \\
& <u^{2}>=\frac{1}{2 \lambda}, \\
& <u^{3}>=0 \\
& <u^{4}>=\frac{3}{4 \lambda^{2}},
\end{aligned}
$$

where $\lambda=\rho /(2 p)$. The coefficient $a$ in Eq.(16) is related to the spatial distribution of conservative variables via

$$
\begin{aligned}
& a_{3}=\frac{4 \lambda_{0}^{2}}{(K+1) \rho_{0}}\left[2(\rho E)_{x}+\left(U_{0}^{2}-\frac{K+1}{2 \lambda_{0}}\right) \rho_{x}-2 U_{0}(\rho U)_{x}\right], \\
& a_{2}=\frac{2 \lambda_{0}}{\rho_{0}}\left[(\rho U)_{x}-U_{0} \rho_{x}\right]-U_{0} a_{3}, \\
& a_{1}=\frac{1}{\rho_{0}} \rho_{x}-U_{0} a_{2}-\frac{1}{2}\left(U_{0}^{2}+\frac{K+1}{2 \lambda_{0}}\right) a_{3},
\end{aligned}
$$

where $\left(\rho_{0}, U_{0}, \lambda_{0}\right)$ are the primitive variables at the cell interface. For the isothermal static gas, the relation reduces to

$$
a_{1}=\frac{1}{\rho_{0}} \frac{\partial \rho}{\partial x}, a_{2}=a_{3}=0 .
$$

The source term in Eq.(15) is

$$
\mathbf{G}_{i}=\int-\phi_{x} f_{u} \psi d u
$$

After integration by parts, this gravitational source can be written as

$$
\begin{aligned}
& G^{\rho}=0, \\
& G^{\rho U}=\rho \phi_{x}, \\
& G^{\rho E}=\rho U \phi_{x}=0 .
\end{aligned}
$$

Then, the UGKS holds the following balance relationships for the mass, momentum and energy. 
1. Mass balance

$$
\begin{gathered}
F_{i-1 / 2}^{\rho}=F_{i+1 / 2}^{\rho}=0, \\
G_{i}^{\rho}=0 .
\end{gathered}
$$

2. Momentum balance

$$
\begin{gathered}
F_{i-1 / 2}=\frac{\rho_{i-1 / 2}}{2 \lambda}, F_{i+1 / 2}=\frac{\rho_{i+1 / 2}}{2 \lambda}, \\
G_{i}^{\rho U}=\rho \phi_{x} .
\end{gathered}
$$

In this situation, we can use the density distribution in Eq.(14) to check the balance relation in cell $i$, i.e.,

$$
\int_{x_{i-1 / 2}}^{x_{i+1 / 2}} G_{i}^{\rho U} d x=\int_{x_{i-1 / 2}}^{x_{i+1 / 2}} \rho \phi_{x} d x=R T\left(\rho_{i+1 / 2}-\rho_{i-1 / 2}\right)=-\left(F_{i-1 / 2}-F_{i+1 / 2}\right) .
$$

3. Energy balance

$$
\begin{gathered}
F_{i-1 / 2}^{\rho E}=F_{i+1 / 2}^{\rho E}=0, \\
G_{i}^{\rho E}=0 .
\end{gathered}
$$

It is clear that the well-balanced relationship in Eq.(15) is satisfied in the unified gaskinetic scheme. The mass, momentum and energy are well kept in the hydrostatic equilibrium state. As we can see, in the continuum flow regime with intensive particle collisions, the collision time tends to zero, and the forcing term in Eq.(16) is a second order term with respect to collision time $\tau$. In other words, the influence of gravitational acceleration on the particle trajectory between successive collisions can be omitted, and the external field only contributes a bulk source on fluid elements.

Generally, the influence of gravity on gas flow has a multiple scale nature, which is related with $\Delta t / \tau$. With the increment of collision time $\tau$, the particle free transport and collision are loosely coupled, and the external force will play a much more significant role in the gas evolution process. If we consider the free molecular limit, i.e., $\tau \gg \Delta t$, the UGKS recovers a free transport process

$$
f_{i+1 / 2, k}=\left\{\begin{array}{l}
f_{i+1 / 2, k^{0}}^{L}+\left(-\left(u_{k}-\phi_{x} t\right) t-\frac{1}{2} \phi_{x} t^{2}\right) \sigma_{i, k^{0}}, \quad u_{k}-\frac{1}{2} \phi_{x} t \geq 0, \\
f_{i+1 / 2, k^{0}}^{R}+\left(-\left(u_{k}-\phi_{x} t\right) t-\frac{1}{2} \phi_{x} t^{2}\right) \sigma_{i+1, k^{0}}, \quad u_{k}-\frac{1}{2} \phi_{x} t<0 .
\end{array}\right.
$$

During the enlarged traveling time between two successive collisions, the particles get acceleration with an enhanced velocity, resulting in a macroscopic mass and energy transport along the direction of external force. There is no a hydrostatic solution anymore, and the non-equilibrium gas evolution may spread to the whole flow field. Under such a condition, it is meaningless to discuss the traditional well-balanced property anymore. In the transition 
regime with modest value of $\Delta t / \tau$, the well-balanced concept becomes a coarse-grained approximation on macroscopic point of view. In any case, however, the gravity effect should be explicitly included in the flux evaluation across a cell interface. Not only for the hydrostatic well-balanced property in the continuum regime, it is even more important to accurately capture the transient evolution due to gravitational effect on the particle trajectory. In the unified gas-kinetic scheme, the gravity effect is taken into account in the flux evaluation in Eq.(2) and Eq.(7), and the scale-dependent flux function ensures the multiscale nature of the algorithm, which is consistent with the gravitational evolution process.

\subsection{Summary of the algorithm}

The numerical algorithm of the well-balanced unified gas-kinetic scheme is the following. The UGKS updates both conservative variables and distribution function in Eq.(12) and Eq.(11). The scale-dependent flux function is determined by the particle distribution function at the interface, which comes from the integral solution of kinetic model equation and is given in Eq.(6) for 1D case and Eq.(9) for 2D case. The gravitational effect on the particle trajectory is explicitly considered into the flux evaluation to model gas evolution process accurately. The detailed numerical procedures for UGKS are given in Table 1.

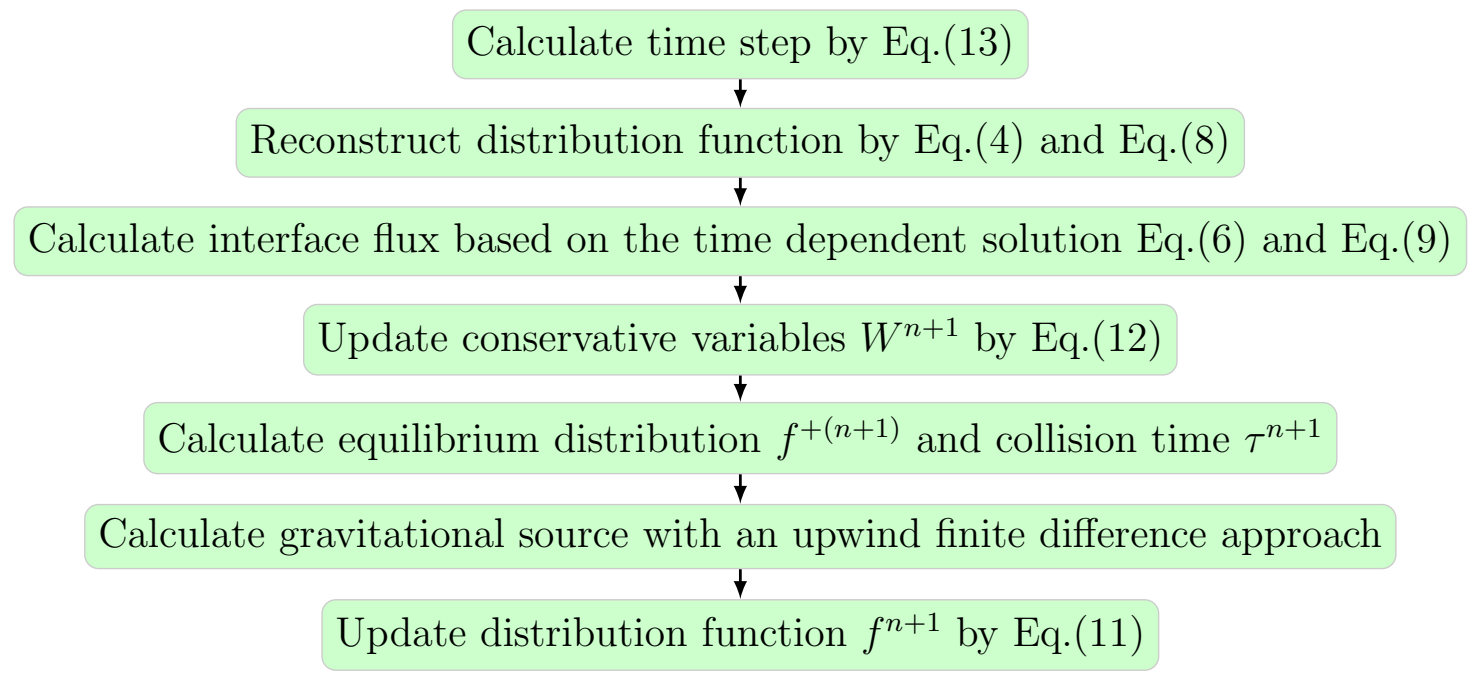

Figure 1: Numerical Algorithm of UGKS.

\section{Numerical experiments}

In this section, we are going to present numerical examples to validate the well-balanced UGKS. In order to demonstrate the capability of the scheme to resolve multi-scale flow physics, simulations from free molecule flow to continuum Euler and NS solutions under the gravitational field will be presented. The flow features in different regimes can be well captured by the unified scheme. For the first time, an interesting non-equilibrium phenomena in the lid-driven cavity case, such as the correlation between the heat flux and 
the gravitational field, will be demonstrated. The Shakhov model is used for the construction of UGKS, and hard sphere (HS) monatomic perfect gas is employed in all test cases.

\subsection{One-dimensional hydrostatic equilibrium solution}

The first case originates from Leveque and Bale's paper [1]. In the simulation, a monatomic ideal gas with $\gamma=5 / 3$ is initially set up with a hydrostatic equilibrium state in the domain $x \in[0,1]$ under the gravitational field $\phi_{x}=-1.0$ pointing towards to the negative $x$-direction,

$$
\rho_{0}(x)=p_{0}(x)=\exp (-x), u_{0}(x)=0 .
$$

The test is for the solution with an added instant perturbation to the initial pressure,

$$
p(x, t=0)=p_{0}(x)+\eta \exp \left(-100(x-5.0)^{2}\right),
$$

where $\eta=0.01$ is a constant. The computational domain is divided into 200 uniform cells and velocity space with 100 discretized velocity points. The Knudsen number for this test case has a value $10^{-4}$, which is basically in the continuum flow regime. The simulation result at $t=0.2$ is presented in Fig. 2. As analyzed in [1,9], operator splitting methods fail to capture such a small perturbation solution, and the effect of gravity should be explicitly considered in the flux evaluation for a well-balanced scheme. It is clear that exact hydrostatic solution under gravity is well preserved by UGKS during the spreading process of the perturbation.

\subsection{Shock tube problem under gravitational field}

The second case is the standard Sod shock tube problem under gravitational field [10, 11]. The computational domain is $x \in[0,1]$, which is divided into 100 cells. The simulation uses monatomic gas with $\gamma=5 / 3$ and non-reflection boundary condition at both ends. The wellbalanced gas-kinetic scheme (GKS) with 1000 mesh points is employed to give the reference solution in the continuum regime $[7,10,11]$.

The initial condition is set as

$$
\begin{gathered}
\rho=1.0, U=0.0, p=1.0, x \leq 0.5, \\
\rho=0.125, U=0.0, p=0.1, x>0.5 .
\end{gathered}
$$

The gravity $\phi_{x}=-1.0$ is in the opposite direction of $x$ axis. The simulation results at $t=0.2$ are presented. In order to present the capability of the unified scheme to simulate flow physics in different flow regimes, we perform the simulations with different reference Knudsen number, such as $\mathrm{Kn}=0.0001, \mathrm{Kn}=0.01$ and $\mathrm{Kn}=1$, which correspond to typical continuum, transition, and free molecular transport. The reference Knudsen number is used to define dynamic viscosity in the reference state via variable soft sphere model (VSS),

$$
\mu_{\text {ref }}=\frac{5(\alpha+1)(\alpha+2) \sqrt{\pi}}{4 \alpha(5-2 \omega)(7-2 \omega)} \mathrm{Kn}_{\mathrm{ref}} .
$$


In this simulation, we choose $\alpha=1.0$ and $\omega=0.5$ to recover a hard sphere monatomic gas. The viscosity for the hard-sphere model is,

$$
\mu=\mu_{r e f}\left(\frac{T}{T_{r e f}}\right)^{\theta}
$$

where $T_{r e f}$ is the reference temperature and $\theta$ is the index related to HS model. In this case we adopt the value $\theta=0.72$. The local collision time is evaluated with the relation $\tau=\mu / p$.

The computational results are presented in Fig. 3. It can be observed that under the gravitational field, the particles inside the tube are "pulled back" in the negative $x$ direction. In comparison with the case without gravity, such as the standard Sod test case, the particle moves towards to the left end of the tube, which results in the increment of density, temperature, pressure, and negative flow velocity.

This test case illustrates the capacity of the unified scheme to simulate flow physics in different regimes under gravitational field. In the continuum regime with $\mathrm{Kn}=0.0001$, the collision time is much less than the time step, which results in the Euler solution and the unified scheme becomes a shock capturing method due to the limited resolution in space and time. The simulation results are consistent with the reference Euler solutions calculated by the GKS, which shows the asymptotic preserving (AP) property of the well-balanced UGKS. With the increment of Knudsen number, the collision time increases and the flow physics changes as well. There is a smooth transion from the Euler solution of the Riemann problem to collisionless Boltzmann solution.

\subsection{Rayleigh-Taylor instability}

This test case comes from [1]. Consider an isothermal static ideal gas with density and pressure satisfying two segmented exponential relations in a two-dimensional polar coordinate $(r, \theta)$,

$$
\rho_{0}(r)=e^{-\alpha\left(r+r_{0}\right)}, p_{0}(r)=\frac{1.5}{\alpha} e^{-\alpha\left(r+r_{0}\right)}, U_{0}=0,
$$

where

$$
\left\{\begin{array} { l } 
{ \alpha = 2 . 6 8 , r _ { 0 } = 0 . 2 5 8 , r \leq r _ { 1 } , } \\
{ \alpha = 5 . 5 3 , r _ { 0 } = - 0 . 3 0 8 , r > r _ { 1 } , }
\end{array} \text { and } \left\{\begin{array}{l}
r_{1}=0.6(1+0.02 \cos (20 \theta)), \text { for density } \\
r_{1}=0.62324965, \text { for pressure }
\end{array}\right.\right.
$$

The external force potential satisfies $d \Phi / d r=1.5$, resulting in a force pointing towards the origin. The initial condition contains a density inversion in the flow region, so there will be an Rayleigh-Taylor instability around the interface under the gravitational field. The fluid motion around the Rayleigh-Taylor unstable interface is expected to be well resolved by the numerical scheme. At the same time, a well-balanced scheme should be able to keep the hydrostatic solution away from the interface.

The computational mesh is a $60 \times 60$ uniform rectangular one, and the velocity space is divided into $20 \times 20$ points. Different reference Knudsen numbers $\mathrm{Kn}=0.0001,0.01$, and 1, are used in the simulation. Here Eq.(19) and Eq.(20) are employed to evaluate the 
relationship between the dynamic viscosity coefficient and the reference Knudsen number, which is the same as the shock tube problem. The specular reflection condition is used at the boundary. The density contours at different output times are presented in Fig. 4. It is clearly demonstrated that the evolution process of the Rayleigh-Taylor instability has different features at different rarefaction conditions. In the continuum regime, as seen in the first row of Fig. 4, the frequent particle collisions prevent the particle penetration and the strong mixing phenomenon happens in the interface region. However, as the Knudsen number increases, the mixing processes speed up through the particle penetration, and the phenomenon of interface instability is much weakened. Fig. 5 shows a scattering plot of density for all cells versus the radius from the center in the polar coordinate. It can be seen that the mixing region in the continuum regime is much narrower than that in the transition and free molecular regimes. Due to the well-balanced property of UGKS, the hydrostatic solution is well kept in the computation, and the mixing process only occurs near the Rayleigh-Taylor unstable interface.

\subsection{Lid-driven cavity flow under gravitational field}

The lid-driven cavity problem is a complex system including boundary effect, shearing structure, heat transfer, non-equilibrium thermodynamics, etc. In this case, we calculate a multi-scale cavity problem under gravity. This test case is an ideal one to validate multi-scale methods.

The square cavity has four walls with $L=1$. The upper wall moves in tangential direction with a velocity $U_{w}=0.15$. The gravity is set to be $\phi_{y}=-1.0,-2.0$ respectively in the negative $y$-direction. The magnitude of gravity $\phi_{y}$ is denoted by $g$. The initial density and pressure are set up with

$$
\rho(x, y, t=0)=\exp \left(\phi_{y} y\right), p(x, y, t=0)=\exp \left(\phi_{y} y\right)
$$

and wall temperature is $T_{w}=2$. Maxwell's accommodation boundary condition is used in the simulation. The Prandtl number of the gas is $\operatorname{Pr}=0.67$. The cavity flow under the same initial and boundary condition with the absence of gravity $\left(\phi_{y}=0.0\right)$ is also simulated for solution comparison. The reference Knudsen number is defined by reference state at bottom of the cavity $\rho_{\text {ref }}=1.0$ and $p_{\text {ref }}=1.0$. The local mean free path $l$ can be evaluated by

$$
l_{\text {local }}=\frac{\mu}{\mu_{\text {ref }}} \sqrt{\frac{p_{\text {ref }}}{p} \frac{\rho_{\text {ref }}}{\rho}} l_{\text {ref }} .
$$

The reference Knudsen number $\mathrm{Kn}=0.075$ is used in the current calculation. The computational domain is divided into $45 \times 45$ uniform cells in the physical space and $28 \times 28$ Gaussian points in the velocity space. Fig. 6 is the solution without gravitational field. Fig. 7 and 8 present the solutions under gravitational field.

In the case under gravitational field, the movement of upper wall and gravitational force are two driving sources for the flow motion in the cavity system. The initial hydrostatic density distribution is perturbed from the upper wall movement. Due to the gravitational force, the density changes significantly along the vertical direction, so is the local Knudsen 
number. In other words, for such a single test case, the gas in the upper and lower parts of the cavity stays in different flow regimes. As demonstrated, even with the viscous heating at the upper wall, the temperature of the gas around the upper surface of the cavity decreases due to the energy exchange among gravitational, kinetic, and internal energy. In comparison with the simulation results without gravitational field in Fig. 6, the heat under gravity transports in the gravitational field direction from the cold (upper) to the hot (lower) regions. The cooling of the upper region may have similar mechanism as the dynamic cooling of the upper atmosphere of the earth. This observation is against Fourier's law, which is also different from the non-equilibrium heat flux due to the rarefaction effect only [16], such as the heat flux shown in Fig. 6. This is the first time that the effect of the gravity on the heat flux has been observed quantitatively. This is a fully non-equilibrium phenomenon. The results show that the heat flux is from the cold to the hot region due to the gravitational effect, which may be used to explain the gravity-thermal instability in astrophysics. Different from the equilibrium thermodynamics, the shift and distortion of the gas distribution function due to the external forcing term provide the dominant mechanism for the non-equilibrium heat flux, especially in the transition flow regime with modest Knudsen number.

The U-velocity and $\mathrm{V}$-velocity distributions at the central vertical and horizontal lines of the cavity as well as the local Knudsen number are presented in Fig. 9 and Fig. 10. As presented in Fig. 9, with the increment of gravitational force, the fluid motion is mostly controlled by gravity. In the case with $g=2$, the local Knudsen number changes significantly from 0.5 at top of the cavity to 0.075 at the bottom of the cavity. A multi-scale flow physics appears inside the cavity. This case clearly demonstrates the capability of the unified scheme to study highly complicated non-equilibrium flow phenomena.

\section{Conclusion}

Gas dynamics under gravitational field has a multiple scale nature with a large variation of gas density in different regions. Based on the direct modeling, a well-balanced unified gas-kinetic scheme under gravitational field has been constructed in this paper for the flow simulation in all regimes. The well-balanced property of the scheme for keeping a hydrostatic equilibrium solution is validated through numerical tests and theoretical analysis. At the same time, due to the multi-scale modeling the UGKS can capture the non-equilibrium flow phenomena associated with the gravitational field. The UGKS is a reliable algorithm for flow simulation from continuum to rarefied one. In the transition regime, for the first time the non-equilibrium flow phenomenon, such as the correlation between the heat flux and gravitational field, has been observed in the cavity case. This scheme may help modeling of a large-scale atmospheric flow around the earth surface, and of the quantitative study of gravity-thermal instability in astrophysical applications. The well-balanced UGKS provides an indispensable tool for the study of the multiple scale non-equilibrium gas dynamics under gravitational field. 


\section{Acknowledgement}

The current research is supported by Hong Kong research grant council (16207715, 16211014, 620813), and National Science Foundation of China (91330203,91530319). 


\section{References}

[1] Randall J LeVeque and Derek S Bale. Wave propagation methods for conservation laws with source terms. In Hyperbolic problems: theory, numerics, applications, pages 609-618. Springer, 1999.

[2] N Botta, R Klein, S Langenberg, and S Lützenkirchen. Well balanced finite volume methods for nearly hydrostatic flows. Journal of Computational Physics, 196(2):539-565, 2004.

[3] Yulong Xing and Chi-Wang Shu. High order well-balanced weno scheme for the gas dynamics equations under gravitational fields. Journal of Scientific Computing, 54(2-3):645-662, 2013.

[4] Kun Xu and Juan-Chen Huang. A unified gas-kinetic scheme for continuum and rarefied flows. Journal of Computational Physics, 229(20):7747-7764, 2010.

[5] Juan-Chen Huang, Kun Xu, and Pubing Yu. A unified gas-kinetic scheme for continuum and rarefied flows ii: multi-dimensional cases. Communications in Computational Physics, 12(03):662-690, 2012.

[6] Juan-Chen Huang, Kun Xu, and Pubing Yu. A unified gas-kinetic scheme for continuum and rarefied flows iii: Microflow simulations. Communications in Computational Physics, 14(05):1147-1173, 2013.

[7] Kun Xu. Direct modeling for computational fluid dynamics: cobnstruction and application of unified gas-kinetic schemes. 2015.

[8] Kun Xu. A well-balanced gas-kinetic scheme for the shallow-water equations with source terms. Journal of Computational Physics, 178:533-562, 2002.

[9] CT Tian, Kun Xu, KL Chan, and LC Deng. A three-dimensional multidimensional gas-kinetic scheme for the navier-stokes equations under gravitational fields. Journal of Computational Physics, 226(2):2003-2027, 2007.

[10] Kun Xu, Jun Luo, and Songze Chen. A well-balanced kinetic scheme for gas dynamic equations under gravitational field. Advances in Applied Mathematics and Mechanics, 2(2):200-210, 2010.

[11] Jun Luo, Kun Xu, and Na Liu. A well-balanced symplecticity-preserving gas-kinetic scheme for hydrodynamic equations under gravitational field. SIAM Journal on Scientific Computing, 33(5):2356-2381, 2011.

[12] Prabhu Lal Bhatnagar, Eugene P Gross, and Max Krook. A model for collision processes in gases. i. small amplitude processes in charged and neutral one-component systems. Physical review, 94(3):511, 1954.

[13] Lowell H Holway Jr. New statistical models for kinetic theory: methods of construction. Physics of Fluids (1958-1988), 9(9):1658-1673, 1966.

[14] EM Shakhov. Generalization of the krook kinetic relaxation equation. Fluid Dynamics, 3(5):95-96, 1968.

[15] Chang Liu, Kun Xu, Quanhua Sun, and Qingdong Cai. A unified gas-kinetic scheme for continuum and rarefied flows iv: Full boltzmann and model equations. Journal of Computational Physics, 314:305-340, 2016.

[16] Benzi John, Xiao-Jun Gu, and David R Emerson. Effects of incomplete surface accommodation on non-equilibrium heat transfer in cavity flow: a parallel dsmc study. Computers \& fluids, 45(1):197-201, 2011. 


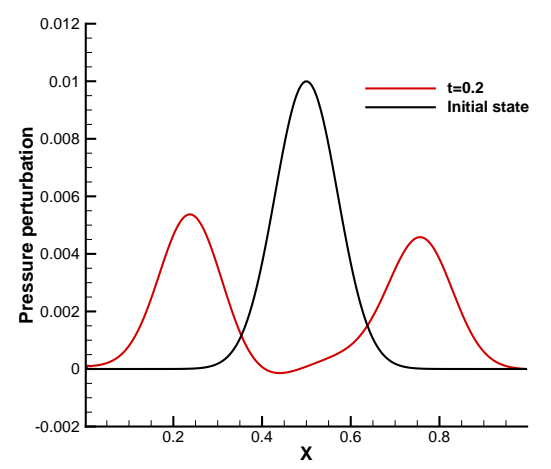

Figure 2: Pressure distribution starting from a perturbation on a hydrostatic equilibrium solution.

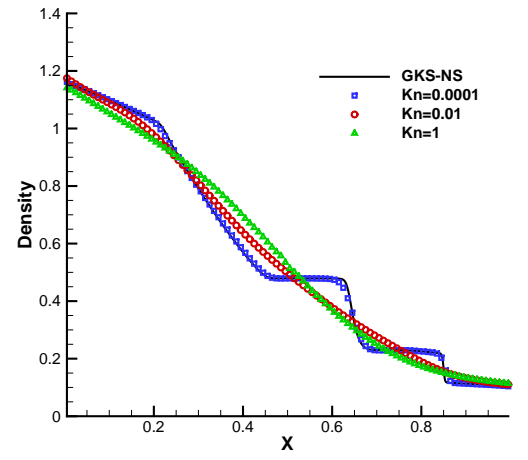

(a) Density

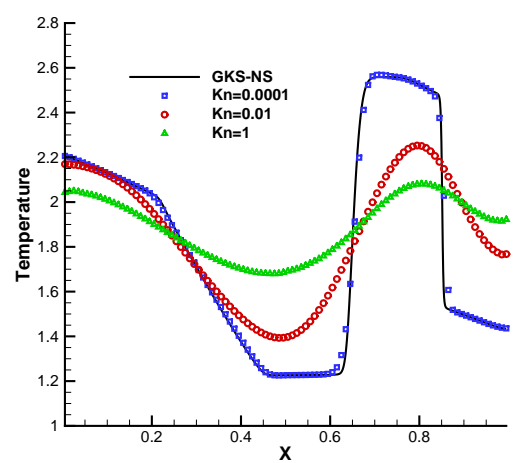

(c) Temperature

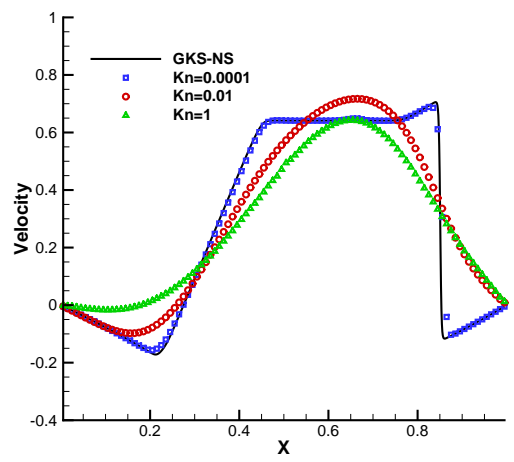

(b) Velocity

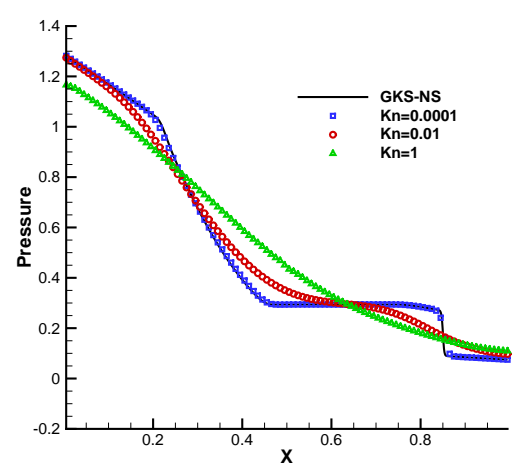

(d) Pressure

Figure 3: Sod test under gravity. 


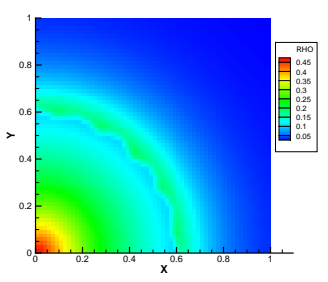

(a) $t=0$

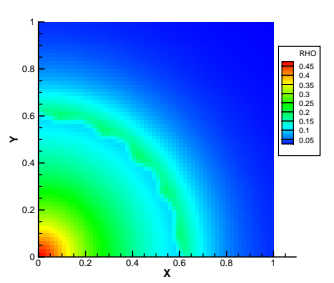

(e) $t=0$

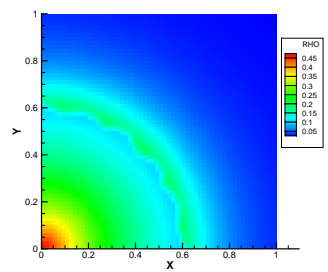

(i) $t=0$

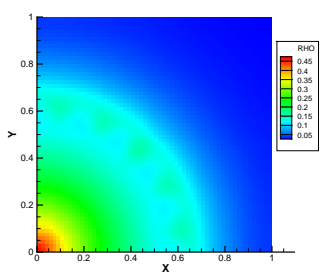

(b) $\mathrm{t}=0.8$

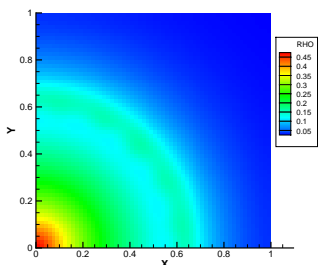

(f) $\mathrm{t}=0.08$

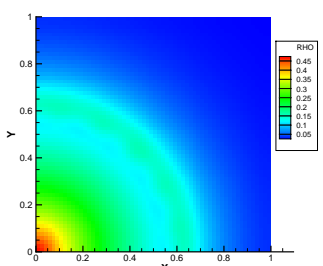

(j) $t=0.08$

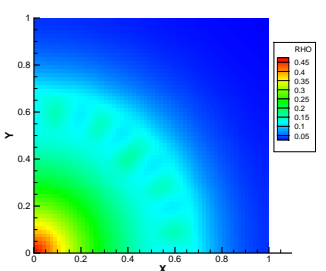

(c) $\mathrm{t}=1.2$

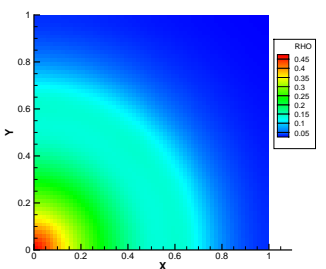

(g) $t=0.16$

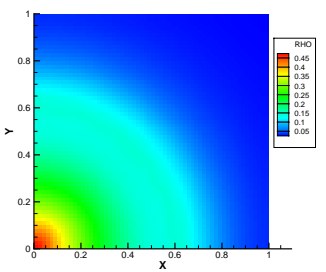

(k) $\mathrm{t}=0.16$

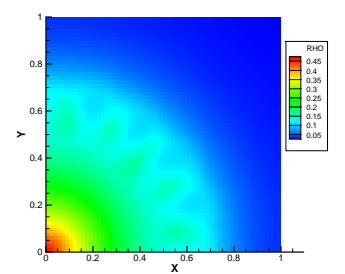

(d) $\mathrm{t}=2.0$

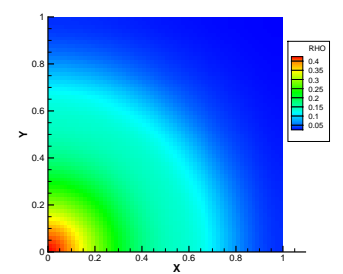

(h) $t=0.24$

Figure 4: Density evolution under gravity at reference Knudsen numbers 0.0001 (1st row), 0.01 (2nd row), 1 (3rd row). 


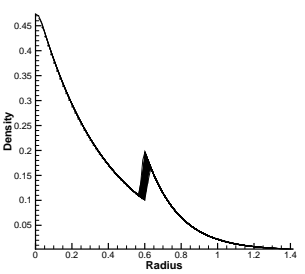

(a) $t=0$

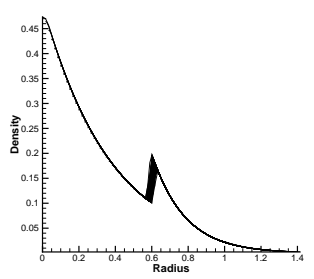

(e) $t=0$

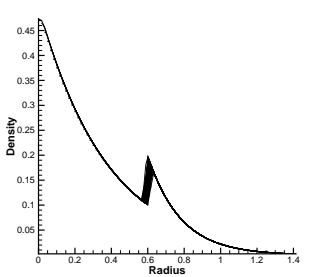

(i) $\mathrm{t}=0$

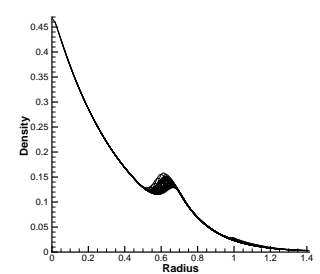

(b) $t=0.8$

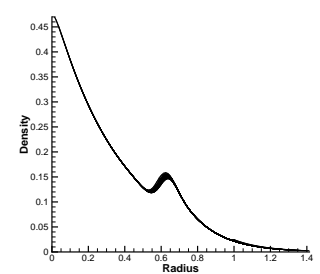

(f) $\mathrm{t}=0.08$

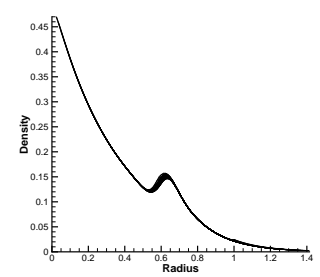

(j) $t=0.08$

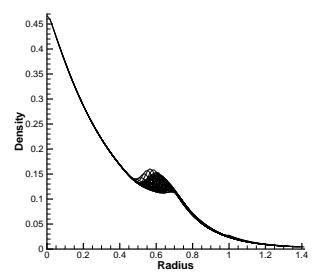

(c) $\mathrm{t}=1.4$

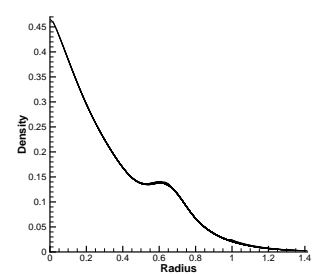

(g) $\mathrm{t}=0.16$

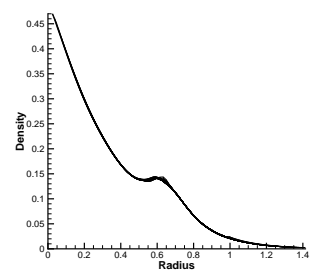

(k) $\mathrm{t}=0.16$

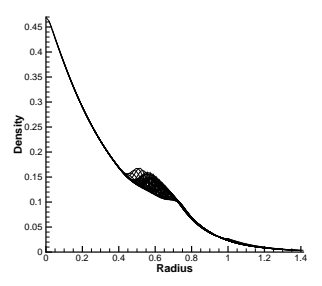

(d) $\mathrm{t}=2.0$

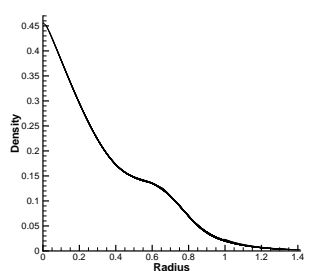

(h) $\mathrm{t}=0.24$

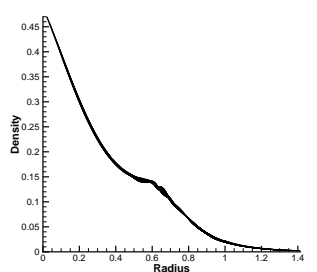

(l) $t=0.24$

Figure 5: Density distribution along the radial direction at reference Knudsen numbers 0.0001 (1st row), 0.01 (2nd row), 1 (3rd row). 


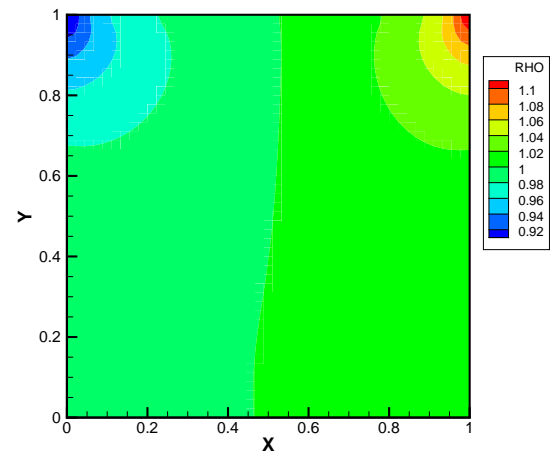

(a) Density

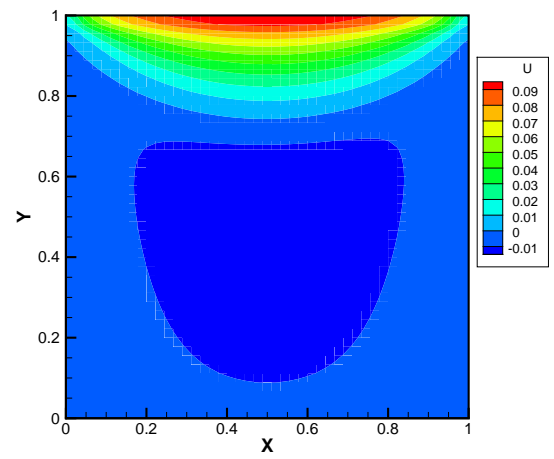

(c) U-velocity

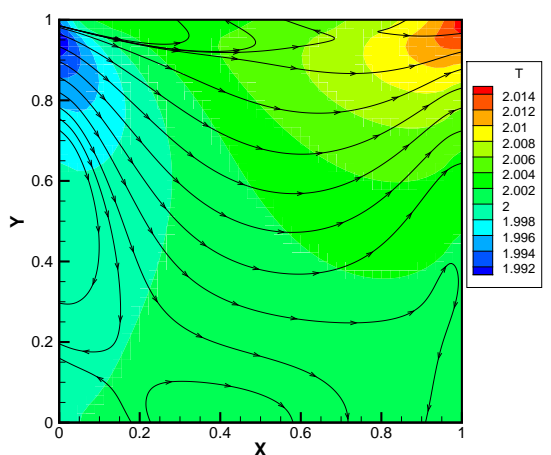

(b) Temperature and heat flux

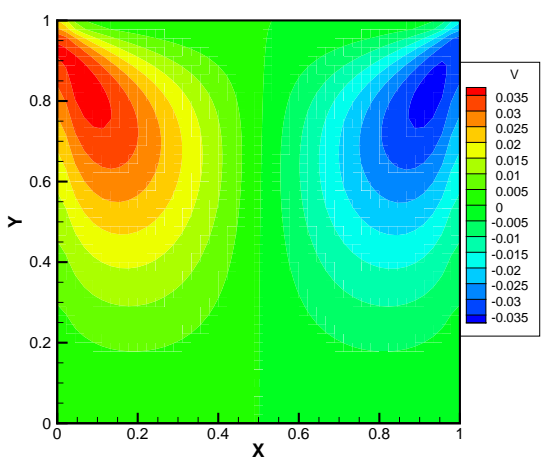

(d) V-velocity

Figure 6: Solutions without gravitational field. 


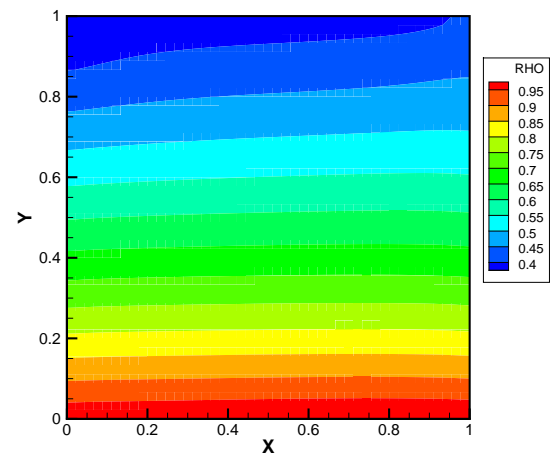

(a) Density

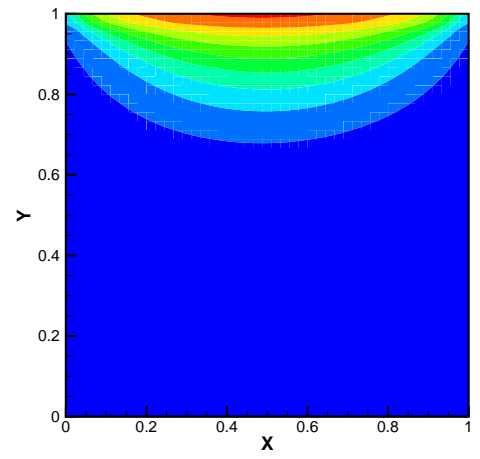

(c) U-velocity

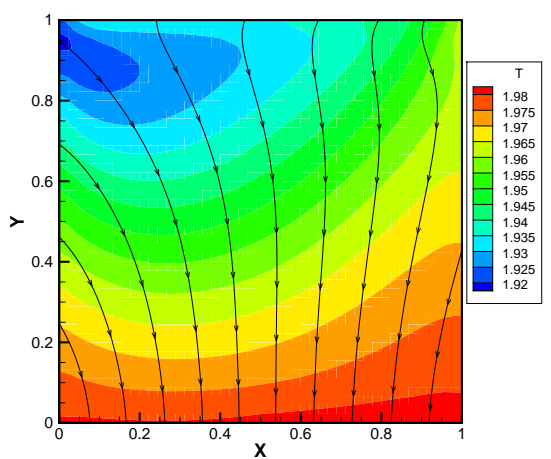

(b) Temperature and heat flux

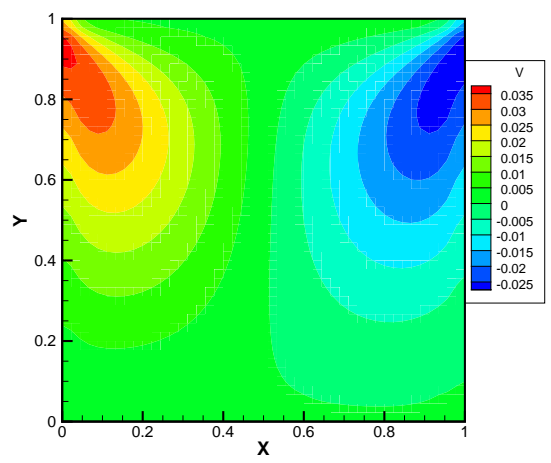

(d) V-velocity

Figure 7: Solutions at $\phi_{y}=-1.0$. 


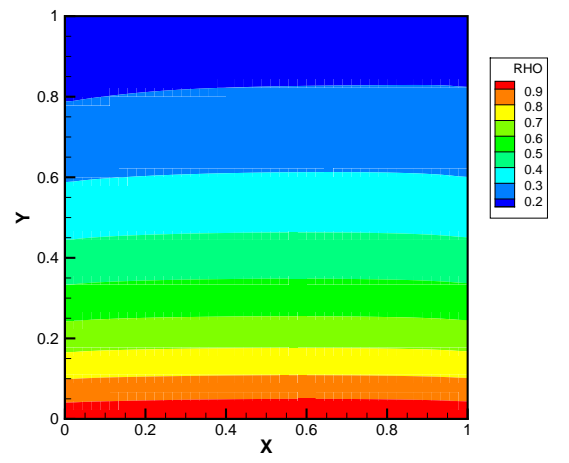

(a) Density

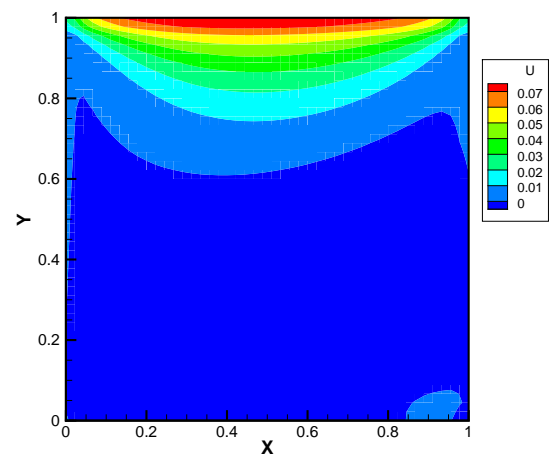

(c) U-velocity

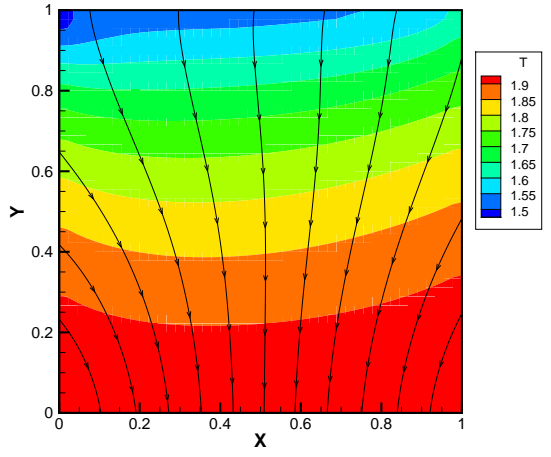

(b) Temperature and heat flux

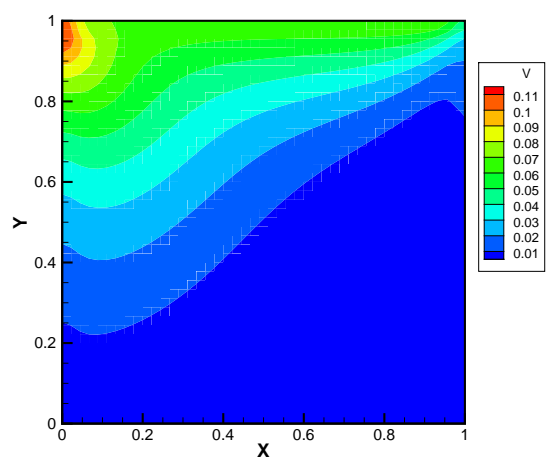

(d) V-velocity

Figure 8: Solutions at $\phi_{y}=-2.0$.
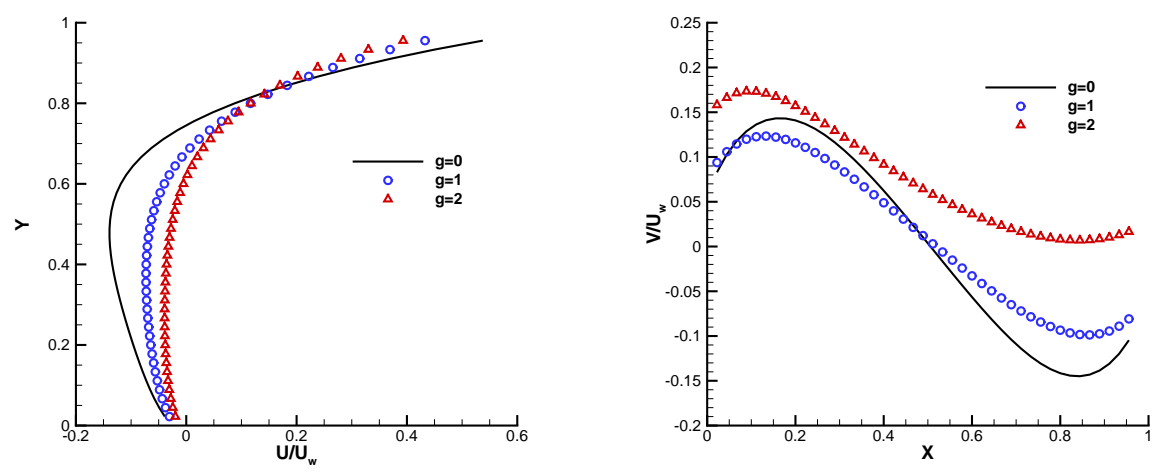

(a) U-velocity along the vertical center (b) V-velocity along the horizontal center line line

Figure 9: U,V velocity along the horizontal and vertical center lines. 


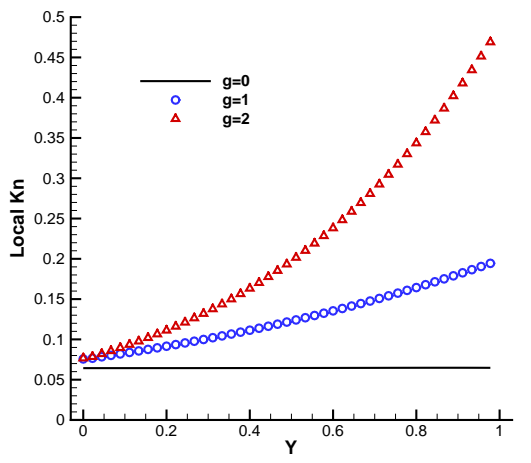

Figure 10: Local Knudsen number along the vertical center line. 